\title{
Der Palmengarten auf dem Fürstlichen Gartenfest auf Schloss Wolfsgarten
}

\author{
Jörg KunZ \& Dirk Ullrich
}

Zum sechsten Mal fand von Freitag, 14., bis Sonntag, 16. September 2012 im Park des Schlosses Wolfsgarten in Langen das Fürstliche Gartenfest statt. Die Fürstlichen Gartenfeste im Herbst in Langen sowie Pfingsten in Eichenzell werden vom Landgrafen Moritz v. Hessen und seinem Team ausgerichtet. Von Anfang an war der Palmengarten mit einem Informationsstand, zusammen mit dem Stand der „Freunde des Palmengartens e.V. - Palmengarten-Gesellschaft", vertreten.

Der September ist ein idealer Monat für zahlreiche Aktivitäten, sodass es oft zu Terminüberschneidungen kommt. In 2012 fanden günstigerweise das Fürstliche Gartenfest und das Herbstfest im Palmengarten nicht wie sonst zeitgleich, sondern an zwei aufeinanderfolgenden Wochenenden statt. Wie immer gab es fürstliches Wetter mit viel Sonnenschein und hohen, fast noch sommerlichen Temperaturen. Jedes Jahr wird das Gartenfest unter ein neues Motto gestellt, 2012 lautete das Sonderthema „Gehölze in Form“.

In diesem Jahr wurde der Stand des Palmengartens besonders aufwendig gestaltet. Dies erforderte natürlich allerlei Vorbereitungen. Tische und Marktschirm, Banner, auszustellende Pflanzen, Steine, Exponate, Flyer, Palmengarten-Infomaterial, Jahresprogramm etc. benötigten unseren LKW sowie Kleitransporter, um nach Langen geschafft zu werden. Die beiden Autoren hatten alles für die Standgestaltung bestens vorbereitet. Am Donnerstag wurde aufgebaut. Aus Felsbrocken und einigen in Form geschnittenen Koniferen wurde ein kleiner asiatisch anmutender Garten aufgebaut. Eine Attraktion waren Hochstämmchen aus Lonicera pileata in Pfeilform. Im Hintergrund wurde es herbstlich mit Chrysanthemen-Gestecken in Gelb und Grün. Farbtöne von Gelb bis Oran-

Abb. 1: DiRk UlLRICH bei der Vorbereitung.
ge-Rot der Tagetes, Kürbisse und Tomaten zauberten ein herbstliches Ambiente. Körbe auf einem Bistrotisch waren gefüllt mit palmengarteneigener Fruchternte. Tatkräftige Unterstützung gab es durch Herbert Billensteiner, der sich um den Stand der Palmengarten-Gesellschaft kümmerte.

Bereits am Freitagvormittag strömten bei dem herrlichen Wetter zahlreiche Pflanzenund Gartenfreunde in den Schlosspark, an den drei Tagen waren es insgesamt rund $21000 \mathrm{Be}$ sucher. Da sich unser Stand in exponierter Lage unterhalb des Messebüros befand, waren wir schon bald ziemlich von Wissbegierigen umlagert. Unsere „Theke“ bot vielseitiges Anschauungsmaterial zum Thema Bäume (in Form) und Holz: Rezente Baumscheiben mit Jahresringen zum Zählen, versteinertes Palmen- und

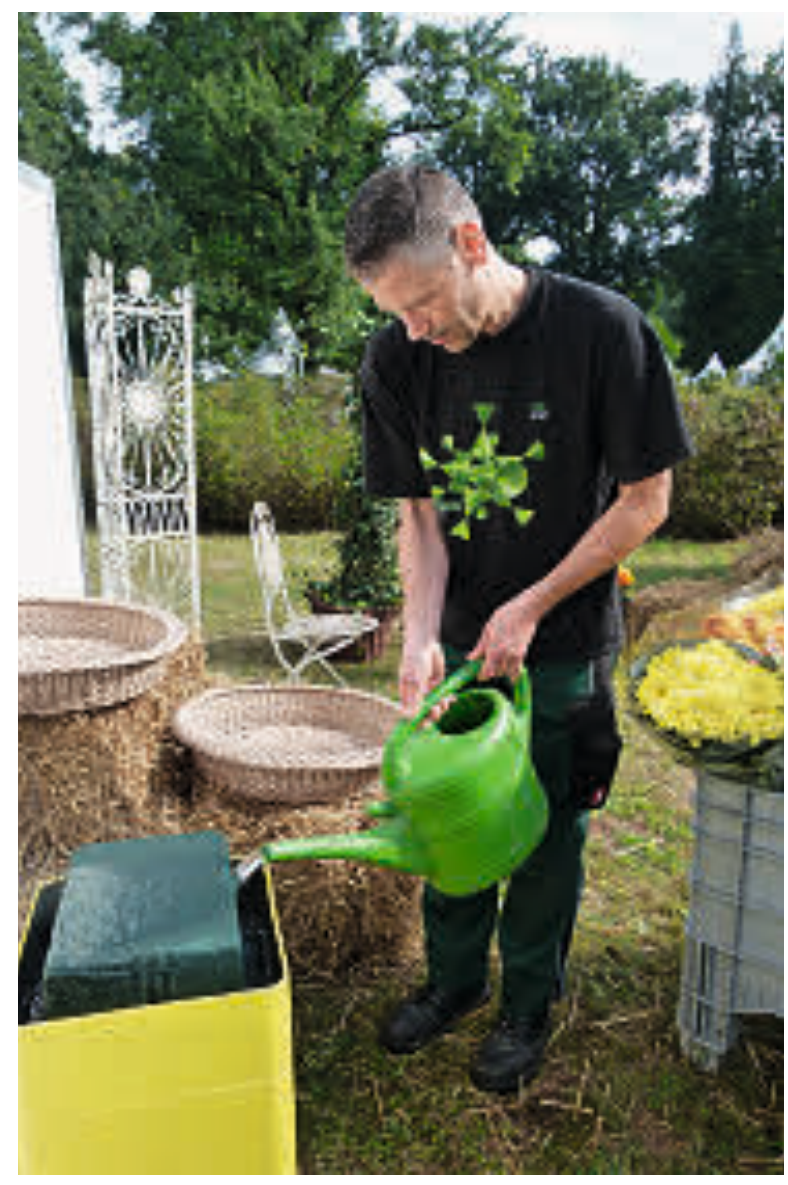




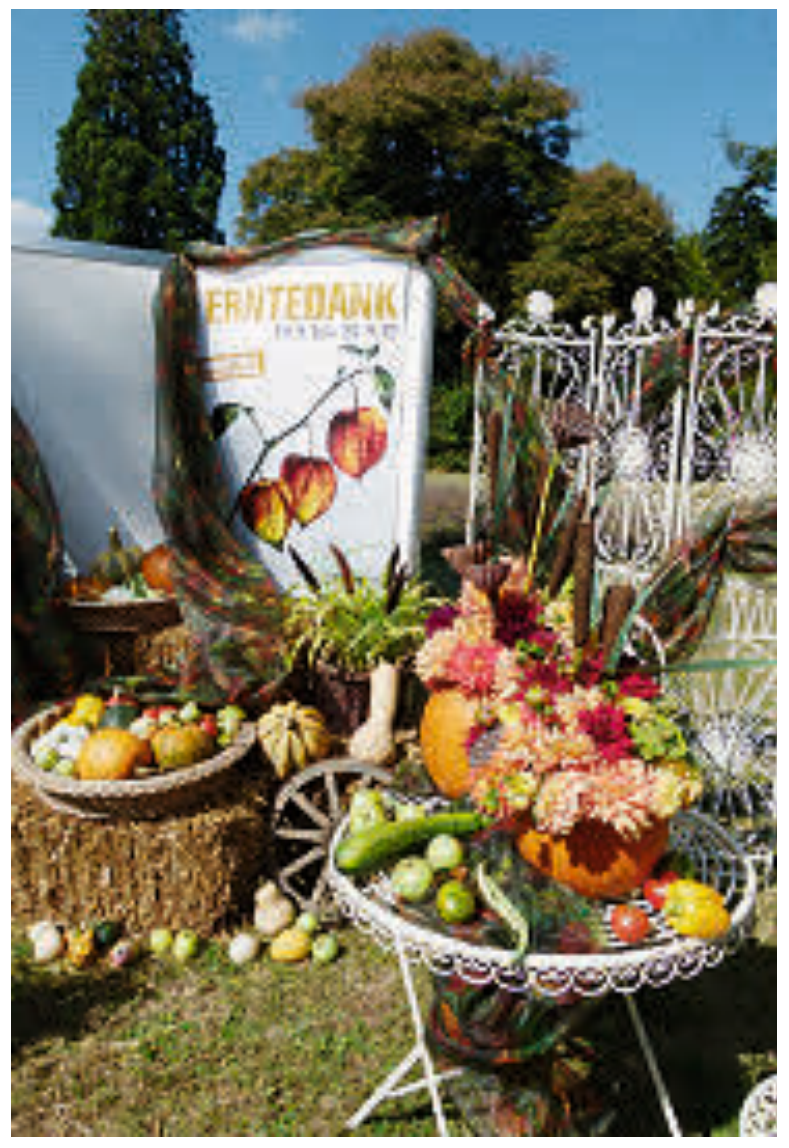

Koniferenholz, diverse kunsthandwerkliche hölzerne Gegenstände, bunt gefüllte Setzkästen mit diversen Baumfrüchten und Koniferenzapfen zum Erraten. Ein Hingucker war auch ein etwa $1,50 \mathrm{~m}$ langes Stück Naturkork einer Korkeiche. Als Mitmachaktion hatten wir einen toten und kahlen Baum an unseren Stand gestellt. Vorbeikommende wurden aufgefordert, auf bereits ausgeschnittene grüne Tonpapierblätter den Namen ihres Lieblingsbaumes zu schreiben. Im Laufe der drei Tage ergrünte der Baum zusehends, Favoriten unter den Bäumen waren Ginkgo und Ahorn. Auch S. K. H. Landgraf Moritz vON Hessen verewigte sich mit einem Blatt, sein Lieblingsbaum ist eine Hickorynuss (Carya). Wer eine "Geheimschrift aus Baumblätterbuchstaben“ richtig löste und die vorbereiteten Rätselblätter ausfüllte, hatte schließlich ein Gedicht von Hermann Hesse über Bäume vor sich liegen.

Die Standbetreuung übernahmen im Wechsel Herbert Billensteiner, Jörg Kunz, Conny Schaan, Peter Schubert, Lolita Sebastian,

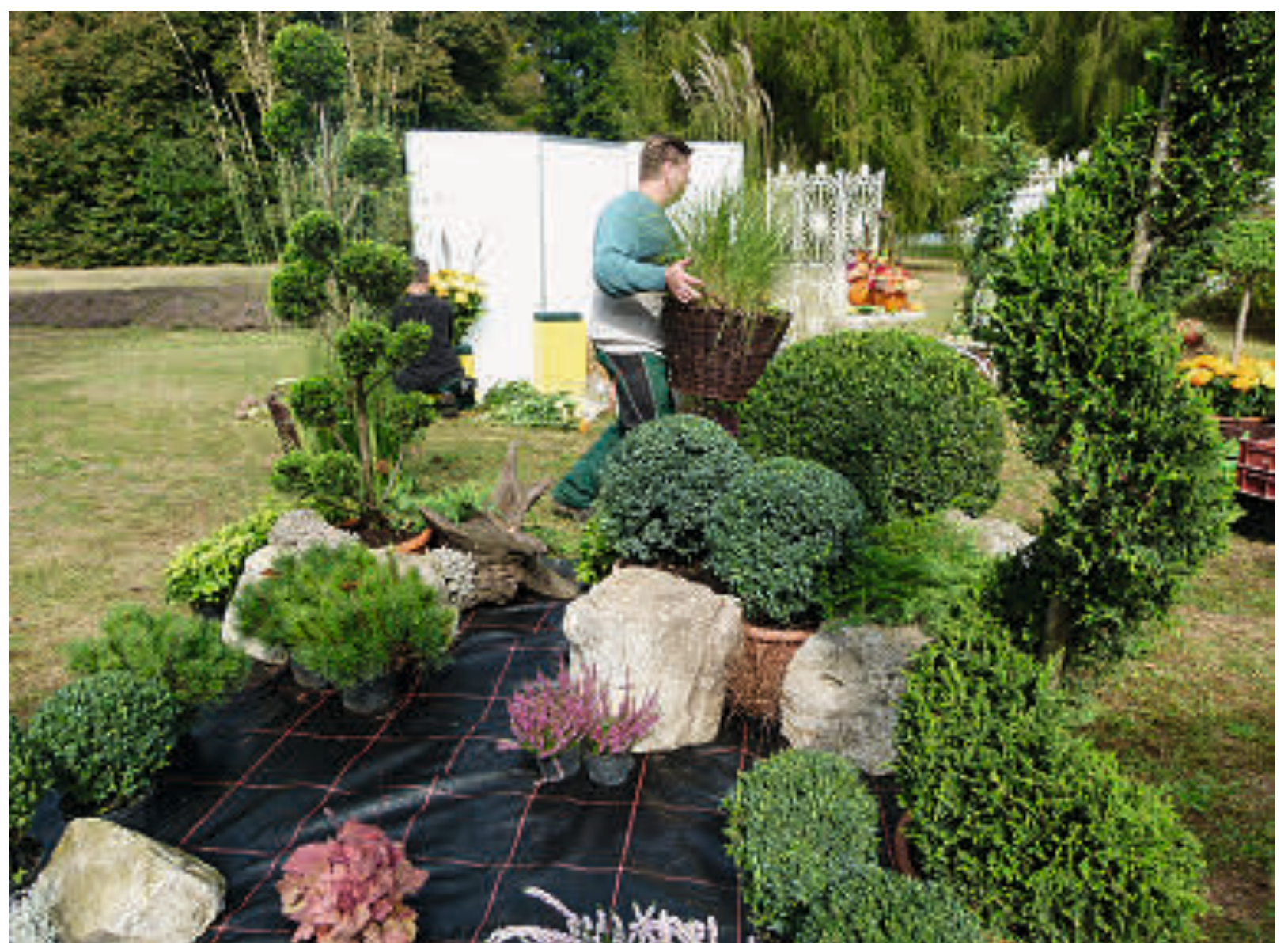




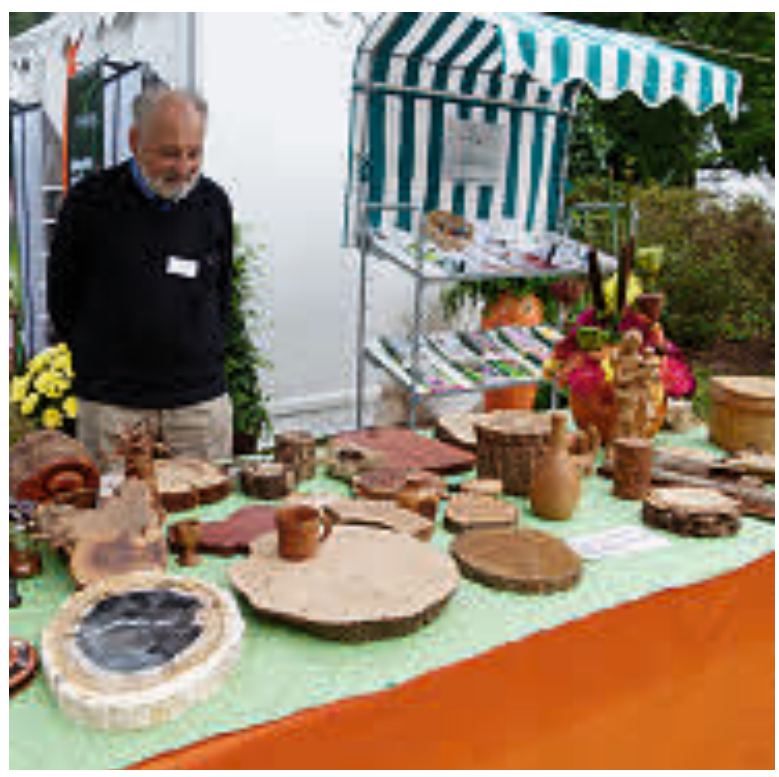

Astrid Steindorf und Hilke Steinecke. Es wurde an allen drei Tagen fast ununterbrochen, informiert, beraten und vor allem Werbung für den Palmengarten und die Palmengarten-Gesellschaft gemacht. Es blieb kaum Zeit, andere Stände mit ihrem z. T. hochwertigen Pflanzenangebot genauer zu erkunden. Stets gibt es viel im Wolfsgarten zu entdecken, neben den Ausstellern z.B. auch die vielen schönen alten Bäume, den japanischen Garten mit der nun komplett renovierten historischen Brücke, das alte Schwimmbad oder das hergerichtete Eibenlabyrinth, das 2012 erstmals wieder der Öffentlichkeit zugänglich gemacht wurde.

Als Jurymitglied für den Botanikpreis war Hilke Steinecke am Freitag zwischen den Ausstellern unterwegs, um die drei Sieger des Botanikpreises auszuloben. Das Juryteam beriet auch S.K.H. Landgraf Moritz von Hessen bei der Wahl des Landgraf- von-Hessen-Preises. Letzteren erhielt Heinrich NiewÖHNER für seine Ulmus davidiana 'Pygmaea'. Es handelt

Abb. 2 (Seite 128 oben): Herbstliche Dekoration.

Abb. 3 (Seite 128 unten): JöRg Kunz gestaltet mit in Form geschnittenen Bäumchen.

Abb. 4 (links): Herbert Billensteiner am Stand.

Abb. 5 (rechts): Holzige Exponate.

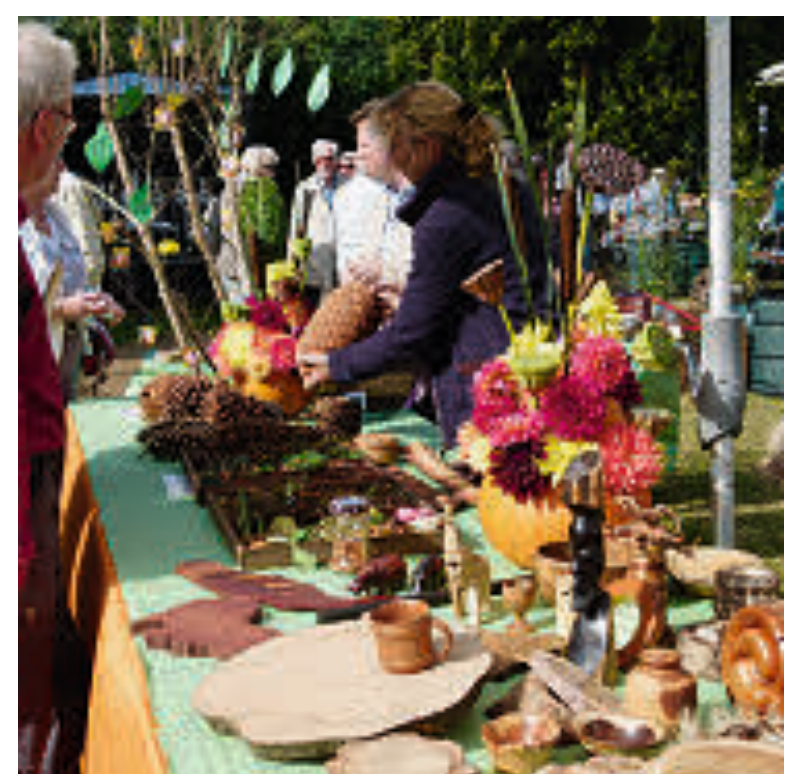

sich um eine nur $30 \mathrm{~cm}$ hohe Zwergform einer chinesischen Ulme, die vermutlich aus einem Hexenbesen entstanden ist und in jeden noch so kleinen Garten passt. „Mit diesem Baum könnte man sich mühelos eine Allee auf dem Küchentisch pflanzen", scherzte S. K. H. DonAtus Prinz von Hessen. Hilke Steinecke durfte am Freitag im Rahmen des Ausstellerabends den goldenen Wolfskopf dem glücklichen Sieger überreichen. Sie ist auch Mitglied des mehrköpfigen Komitees, das sich über Inhalte und Sonderthemen der Fürstlichen Gartenfestes berät. Am Samstagnachmittag führte Iris voN Puttkammer, freiberufliche Mitarbeiterin der Grünen Schule, durch den Park zum Thema „Die Heilkraft der Bäume“.

Der Einsatz aller Beteiligter hat sich wieder gelohnt. Während der vielen anregenden Gespräche haben wir manch einen Besucher erinnert, wieder mal in den Palmengarten zu kommen oder ihn erstmals kennenzulernen. Interessante neue Kontakte wurden geknüpft. Durch derartige Stände werden auch immer wieder neue Mitglieder für die PalmengartenGesellschaft geworben, in 2012 waren es bis zum Herbst 2012 über 120 neue Mitgliedschaften.

Auch in Zukunft wird sich der Palmengarten auf dem Fürstlichen Gartenfest in Langen präsentieren. Für das kommende Jahr lautet das Sonderthema „English Gardening“. 